\title{
La influencia de la Personalidad, la Asignación semanal y los Iguales Antinormativos en el Consumo de Alcohol en la adolescencia
}

\author{
SíGRID GALLEGo MOYA \\ moyam@uji.es \\ LAURA MEZQUITA GUILLAMÓN \\ Imezquit@uji.es \\ LAURA CAMACHO GuERRERO \\ Icamacho@uji.es \\ ANA M. VIRUELA ROYO \\ viruela@uji.es \\ HeLENA ViLLA Martín \\ villa@uji.es
}

\section{Resumen}

El consumo de alcohol se inicia durante la adolescencia, y en él influyen múltiples variables biopsicosociales. A nivel macrosocial se ha estudiado la influencia de diferentes políticas reguladoras (e.g., edad legal de consumo, precios) en el acceso de los jóvenes al alcohol. Sin embargo, la disponibilidad de alcohol a nivel individual (e.g., asignación de la paga semanal) ha recibido una escasa atención. Otra variable ambiental que influye en que los adolescentes consuman alcohol, es el grupo de iguales. Asimismo, variables psicológicas como ser más extravertido e impulsivo jugarían un papel significativo en esta conducta en la adolescencia. El objetivo de este estudio fue analizar la relación entre la personalidad, la asignación de paga semanal y la afiliación con iguales antinormativos en el consumo de alcohol en la adolescencia.

Una muestra de 390 estudiantes de Secundaria respondieron a los cuestionarios JS NEO-S, Deviant Peer Scale (DPS) y AIS-UJI. Los análisis de Regresión Lineal mostraron que los jóvenes más extravertidos, con una mayor asignación de paga semanal o con más amigos antinormativos consumían más alcohol. Además, los adolescentes más extravertidos que, a su vez, tenían una mayor asignación semanal, y los menos responsables que, además, tenían más amigos antinormativos consumían una mayor cantidad de alcohol. Los resultados del presente trabajo muestran el valor predictivo de la personalidad y variables ambientales (paga semanal, afiliación con amigos antinormativos) en el consumo de alcohol. Además, estas variables ambientales parecen moderar el efecto de la personalidad en el consumo de alcohol.

Palabras Clave: Alcohol, Paga Semanal, Personalidad, Adolescentes, Iguales. 


\section{Abstract}

Alcohol use starts during adolescence and is influenced by multiple biopsychosocial variables. The influence of different regulatory policies (e.g., legal drinking age, prices) on youth access to alcohol has been studied to a macro-social level. However, the availability of alcohol on an individual level (e.g., pocket money) has received little attention. Another environmental variable that influences adolescents' alcohol consumption is the peer group. Moreover, psychological variables such as being more extraverted and impulsive play a significant role in this behaviour in adolescence. The aim of this study was to analyse the relationship between personality, pocket money, and affiliation with deviant peers to alcohol consumption in adolescents.

A sample of 390 high school students completed the JS NEO-S, the Deviant Peer Scale (DPS), and the AIS- UJI questionnaires. Linear regression analyses showed that extraverted teenagers, if they had more pocket money or deviant peers, consumed more alcohol. In addition, higher extraversion combined with higher pocket money was related to more alcohol use. This result was also found in lower conscientiousness combined with having deviant peers. These results show the predictive value of personality, pocket money, and affiliation with deviant peers on drinking. Furthermore, environmental variables (pocket money and deviant peers) seem to moderate the effect of personality on alcohol consumption.

Keywords: Alcohol, Pocket money, Personality, Adolescence, peers.

\section{Introducción}

El alcohol es la sustancia psicoactiva más consumida en nuestro país en la etapa de la adolescencia, y en el desarrollo de su consumo están implicadas múltiples variables biopsicosociales que hacen que el estudio de esta conducta resulte muy complejo (Ruipérez, Ibáñez, Villa y Ortet, 2006).

En España, las encuestas estatales del Ministerio de Sanidad, Servicios Sociales e Igualdad muestran que en jóvenes de entre 14 y 18 años, el consumo de alcohol se suele producir en fin de semana y de forma abusiva, que el 35,6 \% de los jóvenes se han emborrachado durante el último mes y que, de éstos, el $66,5 \%$ ha sido por atracón (binge drinking) (ESTUDES, 2010). A grandes rasgos, se indica que la prevalencia de borrachera y el consumo por atracón (o binge drinking) aumentan conforme avanza la edad (ESTUDES, 2010).

En estas mismas encuestas se incide cada vez más en la influencia de aspectos ambientales, como el acceso (ESTUDES, 2010) o el precio del alcohol (Jamison y Myers, 2008; Skidmore y Murphy, 2011), en la instauración del consumo.

A nivel microeconómico también se ha visto que aquellos jóvenes que cuentan con más dinero mostrarían una actitud más favorable al consumo y menos comportamientos saludables (Varela, Marsillas, Isorna y Rial, 2013). No obstante, la disponibilidad de alcohol a nivel individual ha recibido una escasa atención. Solo unos pocos estudios han examinado el papel de la paga o asignación semanal de los jóvenes. Ariza y Nebot (2000) en una muestra de 2140 jóvenes españoles $(37,2 \%$ chicas; media de edad=15,4 años DT=2,1) encontraron que percibir una paga semanal de más de 6 euros (más de 1000 pesetas) influía de manera significativa en el consumo de alcohol de riesgo, tanto para chicos como para chicas (OR 1,8 y 1,6; respec- 
tivamente) únicamente de manera transversal. Por su parte, Lintonen, Kony y Rimpelä (2001) encontraron también un efecto pequeño pero significativo de la asignación de la paga semanal en el consumo de alcohol.

Además, de entre todas las variables ambientales que se asociarían con el consumo de alcohol durante la adolescencia, la presencia de Amigos Antinormativos parece ser de las más relevantes (Borsari y Carey, 2001). Múltiples trabajos indican que pertenecer a un grupo donde los iguales realizan conductas fuera de la norma (p.e. consumir sustancias o venderlas, delitos, agresiones ), aumenta la probabilidad de llevar a cabo comportamientos antisociales (Monahan, Steinberg, Cauffman, 2009; Matsueda y Anderson, 1998; Heinze, Toro y Urberg, 2004) e incluso conductas de riesgo, tales como el consumo de sustancias o relaciones sexuales sin protección (Trucco, Colder y Wieczorek, 2011). Así, se produce una sinergia entre las conductas y actitudes de los adolescentes y sus iguales (Prinstein, Brechwald y Cohen, 2011), que pueden explicarse, entre otros, por procesos de influencia o de selección (Borsari y Carey, 2001).

Finalmente, de entre las variables psicológicas, características de personalidad de elevada impulsividad / desinhibición, baja Amabilidad (-A) y la baja Responsabilidad (-C), se asocian con distintos patrones y variables de consumo de alcohol (p.e., consumo normativo y por atracón, abuso o dependencia) en las distintas etapas del desarrollo (Ibáñez et al., 2008; Kotov, Gamez, Schmidt y Watson, 2010). Mientras que, de forma específica, la extraversión se ha asociado con el inicio del consumo de alcohol en la adolescencia y parece ejercer un papel facilitador de la interacción social en contextos donde está presente el alcohol (Ibáñez et al., 2008).

Por tanto, el objetivo de este estudio fue analizar la influencia de diversas variables psicológicas (la personalidad) y ambientales (paga semanal, presencia de amigos antinormativos), en el consumo de alcohol durante la adolescencia. Nuestra hipótesis era que, además de mostrar efectos simples aditivos, las variables ambientales ejercerían un papel moderador de las variables de personalidad a la hora de predecir el consumo de los adolescentes.

\section{Método}

\section{Muestra y Procedimiento}

La muestra estaba compuesta de 390 estudiantes de Enseñanza Secundaria Obligatoria de centros de la Comunidad Valenciana (España), tanto de zonas urbanas como rurales. El $57,80 \%$ eran chicas y la media de edad eran 15,23 años (DT = ,64) (rango 14 a 17 años). Los cuestionarios se administraron de forma colectiva, motivando a los participantes y asegurándoles la confidencialidad de los resultados.

\section{Instrumentos}

- El AIS-UJI (Ortet y cols., 2002) evalúa el consumo de alcohol y el dinero que perciben los adolescentes con periodicidad semanal ('paga semanal' o 'asignación semanal'). Las Unidades de Bebida Estándar (UBE; Rodriguez-Martos, Gual y Llopis, 1999) se calcularon a partir de una pregunta semiestructurada en la que los adolescentes informaban el número de vasos de vino, cervezas, combinados y licores sin combinar que tomaban durante el fin de semana. El vino y la cerveza se contabilizan como 1 UBE, mientras el combinado y los licores sin combinar ('chupitos') se contabilizaban 
como 2 UBEs (Rodríguez-Martos, Gual y Llopis, 1999). En España una UBE equivale a 10 gramos de alcohol (Llopis, Gual y Rodríguez-Martos, 2000).

- EI JS NEO-S (Ortet, Ibáñez, Moya, Villa, Viruela y Mezquita, 2012) es la adaptación española para adolescentes (12-18 años) del NEO-PI R (Costa y McCrae, 1992; Avia, 1997) y evalúa las cinco dimensiones Neuroticismo $(N)$, extraversión $(E)$, apertura a la experiencia $(\mathrm{O})$, amabilidad $(\mathrm{A})$ y responsabilidad $(\mathrm{C})$ ) y las treinta facetas del Modelo de los Cinco Factores de Costa y McCrae (1992).

- El Deviant Peer Scale (DPS; Gallego, Viruela, Camacho, Mezquita y González, 2011) es una escala de 22 ítems que pregunta a los adolescentes cuántos de los iguales de su grupo de amigos ( $1=$ ninguno; $5=$ todos) han realizado conductas fuera de la norma durante los últimos 6 meses (p.e. robar, asaltar, consumir y/o vender drogas ilegales).

\section{Análisis}

En primer lugar, se calcularon los datos descriptivos y Correlaciones Bivariadas de Pearson. Además, para analizar los efectos directos y la posible existencia de un efecto de moderación o interacción entre las variables, se realizó un Análisis de Regresión Lineal Jerárquica o por pasos. Los análisis se llevaron a cabo con el programa estadístico IBM SPSS statistics versión 21 (IBM, 2012). Todas las puntuaciones de las variables utilizadas para los análisis de este estudio fueron centradas por la media, se les restó el valor medio de la variable, de forma que las puntuaciones más altas equivalen a más del constructo medido (Cooper, 2000).

\section{Resultados}

\section{Análisis Descriptivos y Correlaciones Bivariadas de Pearson}

Las medias en las variables de personalidad, asignación semanal, cantidad de amigos antinormativos y consumo de alcohol en fin de semana se presentan en la tabla final (véase Tabla 1). Ciento sesenta y siete participantes (42,71\%) informaron que no habían consumido nunca alcohol. La media de consumo de alcohol de los participantes que habían informado que habían bebido alcohol fue de 9,00 UBEs (DT= 8,33).

Por otra parte, 255 jóvenes $(65,22 \%)$, percibían dinero de forma semanal ('paga semanal'). La media del dinero que percibían fue de 11,55 euros a la semana (DT $=9,67$ ). Además, 14 participantes indicaron que no tenían ningún amigo que hubiera realizado algún tipo de conducta fuera de la norma, esta cantidad de jóvenes representa el 3,58 \% de la muestra total. 
Tabla 1

Medias y desviaciones típicas de toda la muestra en cada variable, así como medias y desviaciones típicas por género, pruebas $t$ y $d$ de Cohen

\begin{tabular}{lccccc}
\hline & $\begin{array}{c}\text { TODA LA MUES- } \\
\text { TRA }(\mathrm{N}=391)\end{array}$ & $\begin{array}{c}\text { CHICOS } \\
(\mathrm{N}=164)\end{array}$ & $\begin{array}{c}\text { CHICAS } \\
(\mathrm{N}=227)\end{array}$ & $\mathrm{T}$ & $\mathrm{D}$ \\
\hline Neuroticismo (N) & $60,66(14,21)$ & $54,59(13,31)$ & $65,05(13,21)$ & $-7,70^{* * *}$ &,- 79 \\
Extraversión (E) & $90,91(12,44)$ & $90,04(12,65)$ & $91,54(12,27)$ & $-1,18$ &,- 12 \\
Apertura (O) & $67,71(12,02)$ & $64,20(12,35)$ & $70,25(11,13)$ & $-5,06^{* * *}$ &,- 51 \\
Amabilidad (A) & $74,34(10,82)$ & $71,95(10,78)$ & $76,06(10,53)$ & $-3,78^{* * *}$ &,- 39 \\
Responsabilidad (C) & $84,53(15,05)$ & $83,89(15,69)$ & $84,99(14,60)$ &,- 70 &,- 07 \\
Asignación Semanal & $7,53(9,55)$ & $8,08(11,09)$ & $7,14(8,27)$ &, 95 &, 10 \\
Amigos Antinormativos & $32,31(9,19)$ & $34,47(11,23)$ & $30,75(6,99)$ & $4,02^{* * *}$ &, 40 \\
Consumo de alcohol & $5,16(7,72)$ & $6,07(8,06)$ & $4,50(7,42)$ & $1,99^{*}$ &, 20 \\
en fin de semana & & & & & \\
\hline
\end{tabular}

Nota: ${ }^{*} p<, 05,{ }^{* *} p<, 01,{ }^{* * *} p<, 001$. Valores de $d$ de Cohen de $, 20,50$ y ,80, se corresponden con un tamaño del efecto pequeño, medio y grande, respectivamente (Cohen, 1992).

Tabla 2

Correlaciones Bivariadas de Pearson

\begin{tabular}{|c|c|c|c|c|c|c|c|}
\hline & 2. & 3. & 4. & 5. & 6. & 7. & 8. \\
\hline 1. Neuroticismo $(\mathrm{N})$ &,$- 129^{*}$ &, 062 &,$- 126^{*}$ &,$- 337^{\star *}$ & 020 & 029 & ,059 \\
\hline 2. Extraversión (E) & - &, $119^{*}$ &, $192^{* *}$ & ,099 &, $191^{* *}$ &, 030 &, $133^{* *}$ \\
\hline 3. Apertura $(\mathrm{O})$ & & - &, $304^{* *}$ &, $117^{*}$ &,- 076 &,- 080 &,- 082 \\
\hline 4. Amabilidad (A) & & & - &, $311^{* *}$ &,- 038 &,- 071 &,$- 153^{* *}$ \\
\hline 5. Responsabilidad (C) & & & & - &,$- 143^{* *}$ &,- 018 &,$- 125^{\star}$ \\
\hline $\begin{array}{l}\text { 6. Consumo de alcohol en } \\
\text { fin de semana }\end{array}$ & & & & & - &, $197^{* *}$ &, $375^{* *}$ \\
\hline 7. Asignación Semanal & & & & & & - & ,094 \\
\hline 8. Amigos Antinormativos & & & & & & & - \\
\hline
\end{tabular}

Nota: ${ }^{*} p<, 05,{ }^{* *} p<, 01,{ }^{* * *} p<, 001$.

\section{Regresión Lineal}

Los análisis de Regresión Lineal mostraron que tras controlar el efecto de la edad y el género, la Extraversión (E), la Asignación Semanal (AS), los Amigos Antinormativos (AA) y la interacción entre Extraversión y Asignación Semanal, y entre Responsabilidad y Amigos Antinormativos se asociaron de forma significativa con el consumo de alcohol propio en fin de semana (véase Tabla 3). En la Figura 1 y 2 se pueden observar los efectos de interacción entre 
la personalidad y las variables ambientales a la hora de predecir el consumo de fin de semana de los adolescentes.

Tabla 3

Análisis de regresión lineal

\begin{tabular}{|c|c|c|c|c|c|}
\hline \multicolumn{6}{|c|}{ CONSUMO DE ALCOHOL EN FIN DE SEMANA } \\
\hline & & $\mathrm{R}^{2}$ & $\Delta \mathrm{R}^{2}$ & $\beta$ & $\mathrm{t}$ \\
\hline \multirow{3}{*}{ Paso 1} & & 021 & ,021* & & \\
\hline & Edad & & & 086 & $2,056^{*}$ \\
\hline & Género & & &,- 121 & $-2,042^{*}$ \\
\hline \multirow{8}{*}{ Paso 2} & & ,203 & $182^{* * *}$ & & \\
\hline & Neuroticismo $(\mathrm{N})$ & & & ,000 &,- 007 \\
\hline & Extraversión (E) & & & , 136 & $2,821^{* *}$ \\
\hline & Apertura $(\mathrm{O})$ & & &,- 021 &,- 414 \\
\hline & Amabilidad $(A)$ & & & 011 & ,211 \\
\hline & Responsabilidad (C) & & &,- 090 & $-1,733$ \\
\hline & Paga semanal (AS) & & & , 176 & $3,716^{* * *}$ \\
\hline & Amigos Antinormativos (AA) & & & ,325 & $6,664^{* * *}$ \\
\hline \multirow{12}{*}{ Paso 3} & & ,257 &, $054^{* *}$ & & \\
\hline & $\mathrm{N} \times A S$ & & & ,078 & 1,615 \\
\hline & $E \times A S$ & & & 156 & $2,705^{\star *}$ \\
\hline & OxAS & & & ,029 &, 564 \\
\hline & $A \times A S$ & & &,- 018 & - ,302 \\
\hline & $C \times A S$ & & & ,000 & ,006 \\
\hline & $N \times A A$ & & &,- 103 & $-1,860$ \\
\hline & $E \times A A$ & & & ,072 & 1,322 \\
\hline & $O \times A A$ & & & ,044 & ,766 \\
\hline & $A \times A A$ & & &,- 025 &,- 408 \\
\hline & $C \times A A$ & & &,- 158 & $-2,778^{* *}$ \\
\hline & $A S \times A A$ & & &,- 091 & $-1,781$ \\
\hline
\end{tabular}

Nota. ${ }^{*} p<, 05,{ }^{* *} p<, 01,{ }^{* *} p<, 001$ 


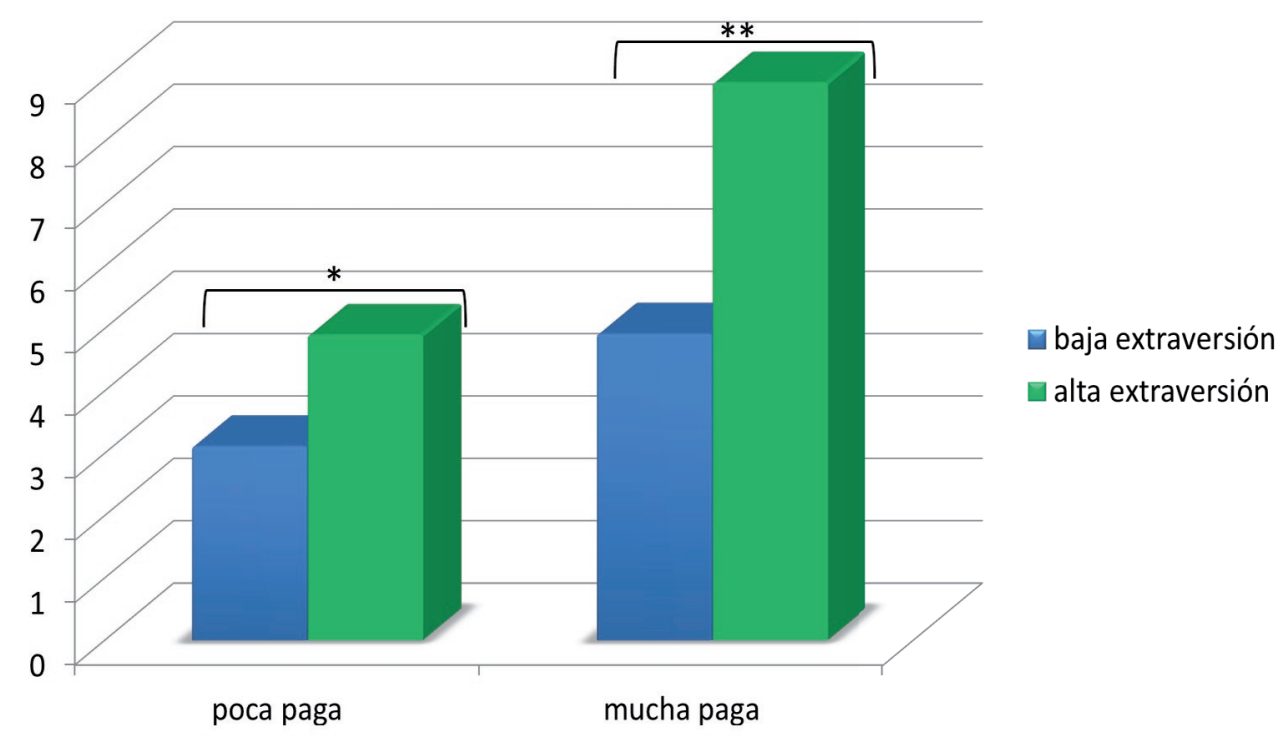

Figura 1. Interacción entre la Extraversión y la Paga semanal en relación al consumo de alcohol (UBES) durante el fin de semana en estudiantes de Secundaria. Nota. ${ }^{*} p<, 05,{ }^{* *} p<, 01,{ }^{* * *} p<, 001$

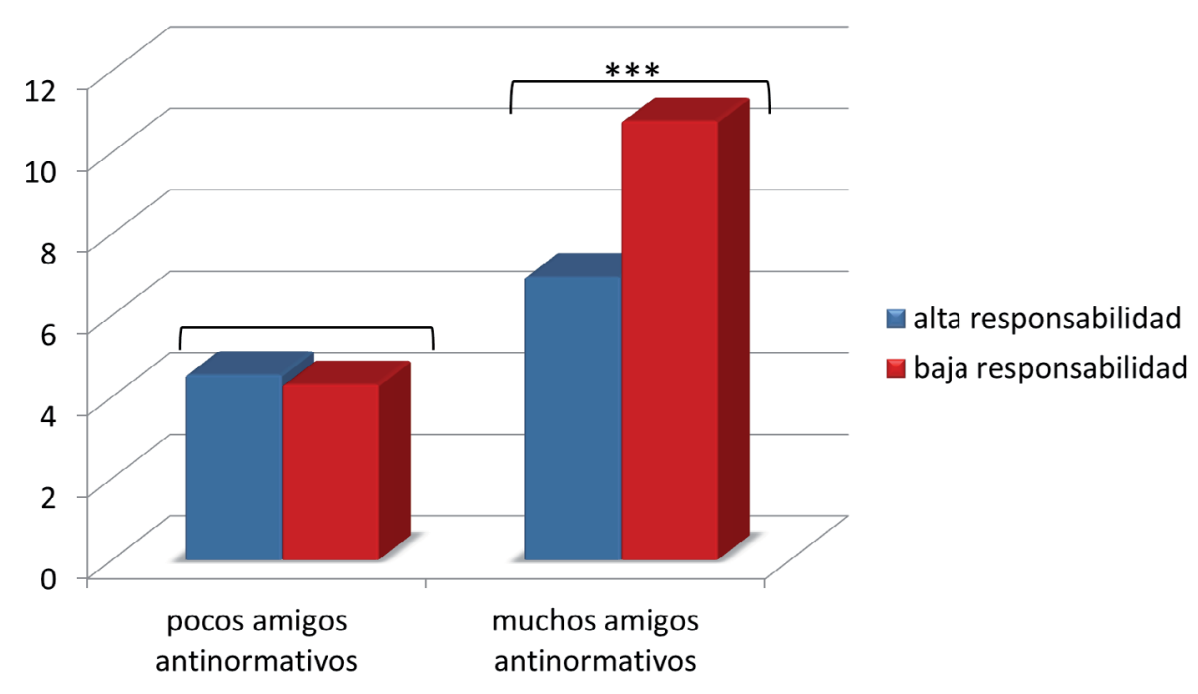

Figura 2. Interacción entre la Responsabilidad y los Amigos Antinormativos en relación al consumo de alcohol (UBES) durante el fin de semana en estudiantes de Secundaria. Nota. ${ }^{*} p<, 05,{ }^{* *} p<, 01,{ }^{* * *} p<, 001$

\section{Discusión y conclusiones}

El objetivo del presente trabajo fue estudiar la relación entre distintas variables psicológicas (personalidad) y ambientales (paga semanal y cantidad de amigos antinormativos) a la hora de predecir el consumo de alcohol en fin de semana de los adolescentes.

Los resultados de la presente investigación mostraron que la asignación semanal, la cantidad de amigos antinormativos y la personalidad (extraversión) se asociaban con el con- 
sumo de alcohol de forma directa. Estos resultados van en la línea de investigaciones previas en las que se encuentra que: a) ser más extravertido facilita el inicio en del consumo de alcohol durante la adolescencia (Ibáñez et al., 2008), seguramente a través de procesos de facilitación social (Ruipérez, et al., 2006; Ibáñez et al., 2008), y que b) disponer de una mayor paga semanal (Ariza y Nebot, 2000; Lintonen et al., 2001), y c) tener un mayor número de amigos que realiza conductas fuera de la norma (Borsari y Carey, 2001; Trucco et al., 2011), se asocia con un mayor consumo de alcohol en esta etapa evolutiva.

En contra de lo esperado, no se encontró asociación directa entre características de desinhibición (-C y/o -A) y el consumo de alcohol (Ibáñez et al., 2008; Kotov et al., 2010). El rol de la desinhibición resultó relevante cuando se estudiaron los efectos de interacción / moderación con las variables ambientales. Así, aquellos adolescentes que eran menos responsables o más desinhibidos y que a su vez tenían más amigos antinormativos consumían mayores cantidades de alcohol durante el fin de semana. Es decir, en nuestra muestra, el ser más desinhibido no sería un factor de riesgo para el consumo de alcohol per se. No obstante, en ciertos contextos en los que se los adolescentes se asociaran con amigos que delinquieran, que consumieran drogas, que robaran..., amigos con mayor probabilidad de patrones de conducta externalizante, la probabilidad de consumir alcohol sería mayor en los más irresponsables en comparación con los adolescentes más responsables.

Por otra parte, tras controlar el efecto de la extraversión y de la asignación de paga semanal se encontró un efecto adicional de interacción entre ambas variables (E X AS) que predecía de forma significativa el consumo de alcohol del fin de semana. Es decir, ser extravertido o tener una mayor paga semanal incrementaría la probabilidad de consumir más alcohol en fin de semana, pero el ser extravertido y a su vez tener una mayor asignación semanal incrementaría de forma exponencial la probabilidad de consumir de los adolescentes.

Finalmente nuestro estudio presenta una serie de limitaciones. Primero, se trata de un estudio transversal $\mathrm{y}$, por tanto, debemos ser cautos a la hora de realizar interpretaciones causales. Segundo, nuestra investigación se centró en el estudio de una variable psicológica (personalidad) y dos variables ambientales (AS y AA) a la hora de predecir el consumo de los adolescentes. Estas variables llegaron a explicar en torno al $26 \%$ de la varianza de la cantidad de alcohol consumida por los adolescentes durante el fin de semana. No obstante, la conducta de consumo de alcohol es una conducta compleja en la que influyen un gran número de factores. La inclusión de otras variables psicológicas (p.e., motivos de consumo de alcohol, expectativas sobre los efectos del alcohol) y ambientales (p.e., estilos educativos parentales, consumo de los amigos, consumo del mejor amigo, etc.) hubiese incrementado el porcentaje de varianza explicada del consumo de fin de semana. Tercero, aunque la cantidad de alcohol consumida es una de las medidas más válidas y utilizadas para evaluar el consumo, la evaluación de la frecuencia de consumo, el consumo por atracón, número de borracheras o problemas asociados con el consumo, hubiese dado una visión más integradora del consumo de alcohol en esta población.

En resumen, el presente trabajo muestra la relevancia de la personalidad, la paga semanal y la afiliación con iguales antinormativos con el consumo de fin de semana de los adolescentes. Además, muestra distintos mecanismos de influencia de estas variables en el consumo (directos, moderación / interacción). Estos hallazgos resultan especialmente relevantes, ya que, hasta donde llega nuestro conocimiento, se trata del primer estudio que explora interacciones entre la personalidad y la paga semanal y la conducta antinormativa de los iguales en relación al consumo de alcohol. Los resultados de la presente investigación resultarán de relevancia para posibles programas de prevención y tratamiento del consumo de alcohol en adolescentes. 


\section{Referencias bibliográficas}

Ariza, C. y Nebot M. (2000). Factors associated with problematic alcohol consumption in schoolchildren. Journal of Adolescent Health, 27, 425-433.

Avia, M. D., Sanz, J., y Sánchez-Bernardos, M. L. (1997). Versión española del Inventario de Personalidad NEO-Revisado (NEO PI-R) de Paul T. Costa y Robert R. McCrae (1992). Versión experimental. Manuscrito no publicado. Facultad de Psicología, Universidad Complutense de Madrid.

Bauman, K. E. y Ennett, S. T. (1996). On the importance of peer influence for adolescent drug use: Commonly neglected considerations. Addiction, 91(2), 185-198.

Borsari, B. y Carey, K. B. (2001). Peer influences on college drinking: A review of the research. Journal of Substance Abuse, 13(4), 391-424.

Cohen, J. (1992). A power primer. Psychological Bulletin, 112, 155-159.

Cooper, M. L., Agocha, V. B. y Sheldon, M. S. (2000). A motivational perspective on risky behviors: the role of personality and affect regulatory processes. Journal of Personality, 68, 1059-1088.

Costa, P. T. y McCrae, R. R. (1992). Revised NEO Personality Inventory (NEO PI-R) and NEO FiveFactor Inventory (NEO-FFI). Professional manual. Odessa, FL: Psychological Assessment Resources.

Gallego, S., Viruela, A., Camacho, L., Mezquita, L. y González, J. A. (2011). Adaptación española del cuestionario de las conductas antinormativas de los amigos (DPS) en adolescentes. Forum de recerca, 16, 1039-1048.

Grau, E. y Ortet, G. (1999). Personality traits and alcohol consumption in a sample of non-alcoholic women. Personality and Individual Differences, 27, 1057-1066.

Heinze, H. J., Toro, P. A. y Urberg, K. A. (2004). Antisocial behaviour and affiliation with deviant peers. Journal of clinical child and adolescent psychology, 33, 336-346.

Ibáñez, M. I., Ruipérez, M. A., Villa, H., Moya, J. y Ortet, G. (2008). Personality and alcohol use. En G. J. Boyle, G. Matthews y D. H. Saklofske (eds.), Handbook of Personality Theory and Testing. New York: Sage.

IBM Corp. Released (2012). IBM SPSS Statistics for Windows, version 21.0. Armonk, NY: IBM Corp.

Jamison, J. y Myers, L. B. (2008). Peer-group and price influence students drinking along with planned behaviour. Alcohol y Alcoholism, 43, 492-497.

Kotov, R., Gamez, W., Schmidt, F. y Watson, D. (2010). Linking "big" personality traits to anxiety, depressive and substance use disorders: a meta-analysis. Psychological Bulletin, 136, 768-821.

Lintonen, T. P., Konu, A. I. y Rimpelä, M. (2001). Identifying potential heavy drinkers in early adolescence. Heatlh Education, 101, 159-168.

Llopis Llácer, J. J., Gual Solé, A. y Rodríguez-Martos Dauer, A. (2000). Registro del consumo de bebidas alcohólicas mediante la unidad de bebida estándar. Diferencias geográficas. Adicciones, 12, 11-19.

Matsueda, R. L. y Anderson, K. (1998). The dinamics of delinquent peers and delinquent behavior. Criminology, 36, 269-308.

Monahan, K. C., Steinberg, L. y Cauffman, E. (2009). Affiliation with antisocial peers, susceptibility to peer influence, and desistance from antisocial behavior during the transition to adulthood. Developmental Psychology, 45(6), 1520-1530.

Ortet, G., Ibáñez, M. I., Moya, J., Villa, H., Viruela, A. y Mezquita, L. (2012). Assessing the five factors of personality in adolescents: The junior version of the Spanish NEO-PI-R. Assessment, 19, 114-130 
Prinstein, M. J., Brechwald, W. A. y Cohen G. L. (2011). Susceptibility to peer influence: Using a performance-based measure to identify adolescent males at heightened risk for deviant peer socialization. Developmental Psychology, 47, 1167-1172

Skidmore, J. R. y Murphy, J. G. (2011). The effect of drink price and next day responsibilities on college student drinking: a behavioral economic analysis. Psychology of addictive behaviors, 25, 57-68.

Rodríguez-Martos, A., Gual, A., y Llopis, J. J. (1999). La unidad de bebida estándar como registro simplificado del consumo de bebidas alcohólicas y su determinación en España, Medicina Clínica, 112(12), 446-450.

Ruipérez, M. A., Ibáñez, M. I., Villa, H. y Ortet, G. (2006). Factores biopsicosociales en el consumo de alcohol. En L. A. Oblitas (ed.), Altlas de Psicología de la Salud. Bogotá: PSICOM editores.

Trucco, E. M., Colder, C. R., y Wiezczorek, W. F. (2011). Vulnerability to peer influence: a moderated mediation study oh early adolescent alcohol use initiation. Addictive Behaviors, 36, 729-736.

Varela, J., Marsillas, S., Isorna, M. y Rial, A. (2013). El papel de las actitudes, las percepciones y el dinero disponible en el consumo de drogas en adolescentes. Health and Addictions, $13,67-78$. 